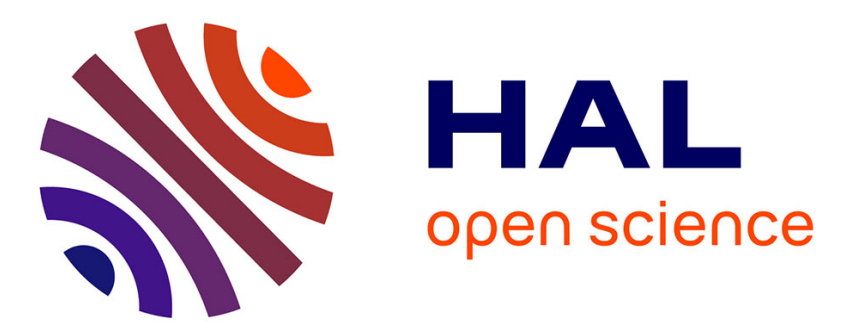

\title{
Observation of the $\delta$ to $\epsilon$ Zr-hydride transition by in-situ synchrotron X-ray diffraction
}

T. Maimaitiyili, Axel Steuwer, Jakob Blomqvist, Christina Bjerkén, Matthew S. Blackmur, Olivier Zanellato, J. Andrieux, Fabienne Ribeiro

\section{- To cite this version:}

T. Maimaitiyili, Axel Steuwer, Jakob Blomqvist, Christina Bjerkén, Matthew S. Blackmur, et al.. Observation of the $\delta$ to $\epsilon$ Zr-hydride transition by in-situ synchrotron X-ray diffraction. Crystal Research and Technology, 2016, 51 (11), pp.663-670. 10.1002/crat.201600234 . hal-02271709

\section{HAL Id: hal-02271709 \\ https://hal.science/hal-02271709}

Submitted on 27 Aug 2019

HAL is a multi-disciplinary open access archive for the deposit and dissemination of scientific research documents, whether they are published or not. The documents may come from teaching and research institutions in France or abroad, or from public or private research centers.
L'archive ouverte pluridisciplinaire HAL, est destinée au dépôt et à la diffusion de documents scientifiques de niveau recherche, publiés ou non, émanant des établissements d'enseignement et de recherche français ou étrangers, des laboratoires publics ou privés. 


\title{
Observation of the $\delta$ to $\varepsilon \mathrm{Zr}$-hydride transition by in-situ synchrotron $X$-ray diffraction
}

\author{
T. Maimaitiyilil,*, A. Steuwer ${ }^{2}$, J. Blomqvist', C. Bjerkén', M.S. Blackmur ${ }^{3}$, O. Zanellato ${ }^{4}$, \\ J. Andrieux ${ }^{5}$, and F. Ribeiro ${ }^{6}$
}

We investigate the formation and dissolution of hydrides in commercially pure zirconium powder in-situ using highenergy synchrotron $\mathrm{X}$-ray radiation. Experimental results showed a continuous phase transition between the $\delta$ and $\varepsilon$ zirconium hydride phases with indication of a second order phase transformation.

\section{Introduction}

Because of their good mechanical properties, excellent corrosion resistance, and a low thermal neutron absorption cross section, zirconium ( $\mathrm{Zr}$ ) alloys have found widespread use as fuel cladding material in nuclear industry [1-4]. Depending on type of nuclear reactor, the fuel cladding is in contact with light $\left(\mathrm{H}_{2} \mathrm{O}\right)$ or heavy water $\left(\mathrm{D}_{2} \mathrm{O}\right)$. During reactor operation these $\mathrm{Zr}$-alloys undergo some corrosion and a fraction of the released hydrogen will diffuse into the alloy. At high temperature $\mathrm{Zr}$ alloys have significantly higher solubility of hydrogen than at room temperatures [5] and Zr-hydrides will form as soon as hydrogen concentration reaches the temperature-sensitive solubility limit. The formation of $\mathrm{Zr}$-hydrides is considered to be a major cause of embrittlement and is also involved in the delayed hydride cracking mechanism [6-8]. The underlying model Zr-H system is still subject of considerable research, in particular the concerning the nature of the individual phases and the transformations. To the best of our knowledge there has not been any in-situ hydrogen charging studies performed on commercial grade $\mathrm{Zr}$ powder and transformation have not been recorded with a single experimental arrangement. In this paper, we report our observation of the transformation between $\delta$ and $\varepsilon$-hydrides by in-situ synchrotron X-ray diffraction studies.

\section{Crystallography of the Zr-H system}

Depending on hydrogen concentration, temperature and cooling rate, three distinct hydride phases have been observed at various temperatures [1-4]. Phases reported in the $\mathrm{Zr}-\mathrm{H}$ system include parent hexagonal close packed (HCP) Zr phase known as $\alpha$-Zr phase, which is of Mg structure type (P63/mmc, $a=3.2316 \AA$, $c=5.1475 \AA$ A); a non-stoichiometric face centered cubic (FCC) $\delta-\mathrm{ZrH}_{x}$ phase, which is of $\mathrm{CaF}_{2}$ structure type (Fm-3m, $a=4.7783 \AA$ ) with hydrogen concentration interval $1.4<x<1.7$; the face centered tetragonal (FCT) $\varepsilon-\mathrm{ZrH}_{x}$ phase with $\mathrm{ThH}_{2}$ structure (I4/mmm, $a=4.9689$, $c=4.4479 \AA$ A), and the stoichiometric FCT ZrH known as $\gamma$ - ZrH phase with structure type $\mathrm{ZrH}(\mathrm{P} 42 / \mathrm{n}, a=$ $4.592 \AA, c=4.970 \AA$ ̊̊). Basic crystallographic data of all these reported phases are tabulated in Table 1. In addition to aforementioned Zr-hydride phases, in 2008 Zhao et al. [9] reported another metastable Zr-hydride phase in Zircaloy-4 which contains small amounts of hydrogen. According to their analysis, this new phase is fully coherent with the $\alpha$-Zr matrix and exists in a hydrogen concentration interval of $0.25 \leq x \leq 0.5$. They named this

\footnotetext{
* Corresponding author: e-mail: tuerdi.maimaitiyilia)mah.se, Phone: +46762945 402, Fax:

1 Materials Science and Applied Mathematics, Malmö University, Nordenskiöldsgatan 1, 20506 Malmö, Sweden

2 Nelson Mandela Metropolitan University, Gardham Avenue, 6031 Port Elizabeth, South Africa

3 Materials Performance Centre, School of Materials, Manchester University, M17HS, Manchester, United Kingdom

4 PIMM, Ensam - Cnam - CNRS, 151 Boulevard de l'Hôpital, 75013 Paris, France

5 European Synchrotron Radiation Facility, 6 rue J Horowitz, 38043 Grenoble, France

6 Institut de Radioprotection et Sûreté Nucléaire, IRSN, BP3 13115 Saint-Paul Lez Durance, France
} 


\begin{tabular}{|c|c|c|c|c|c|c|c|}
\hline Phase & Structure & Space group & $a[\AA]$ & $c[\AA]$ & Temp. $\left[{ }^{\circ} \mathrm{C}\right]$ & Ref. & ICSD No. \\
\hline \multirow[t]{3}{*}{$\alpha(\mathrm{Zr})$} & $\mathrm{HCP}$ & $\mathrm{P} 63 / \mathrm{mmc}$ & 3.2316 & 5.1475 & 25 & [1] & 76042,164572 \\
\hline & & & $3.24205(3)$ & $5.16643(9)$ & 25 & CS & - \\
\hline & & & $3.25730(3)$ & $5.19884(7)$ & $25^{*}$ & & \\
\hline \multirow[t]{2}{*}{$\delta\left(\mathrm{ZrH}_{1.66}\right)$} & FCC & $\mathrm{Fm}-3 \mathrm{~m}$ & 4.7783 & 4.7783 & 20 & [1] & 164605,56198 \\
\hline & & & $4.78395(3)$ & $4.78395(3)$ & $25^{*}$ & CS & - \\
\hline \multirow[t]{2}{*}{$\varepsilon\left(\mathrm{ZrH}_{2}\right)$} & FCT & $14 / \mathrm{mmm}$ & 4.9689 & 4.4497 & 20 & [1] & 656539,638555 \\
\hline & & & $4.97421(4)$ & $4.52438(6)$ & 300 & CS & - \\
\hline
\end{tabular}

*after heat treatment.; $\mathrm{CS}=$ Current study

new phase as $\zeta$ and found that it belongs to the trigonal crystal system with space group P3-m1 $(a=3.27 \AA$, $c=10.83 \AA$ ). However, this new phase have not been confirmed by others and we also did not observed it during our studies. Therefore, we will not discuss it in this paper.

Despite the fact that Zr-hydrides have been studied for several decades, the basic nature and mechanisms of hydride formation and transformation are not yet fully understood, owing to high diffusivity of hydrogen, structural similarities of various phases, extended hydrogen concentration interval of hydride phases and influence of other impurity elements in the $\mathrm{Zr}-\mathrm{H}$ system $[1,2,4$, 10-13].

Regarding the hydrogen rich $\delta$ - to $\varepsilon-\mathrm{ZrH}_{\mathrm{x}}$ transformation, it is still debated at what precise hydrogen concentration [14-16], at what temperature and which kind of transformation order $[15,17-22]$ the FCC $\delta$ - $\mathrm{ZrH}_{\mathrm{x}}$ transforms into the FCT $\varepsilon-\mathrm{ZrH}_{x}$ and vice versa.

Based on ab-initio calculation, Ivashchenko et al. [18] concluded that the cubic-to-tetragonal phase transition in $\mathrm{ZrH}_{2}$ is of "first-order which is close to the secondorder phase transition". In agreement with Ivashchenko, Cantrell et al. [19] also proposed a pseudo-martensitic transition that is of first order with a very narrow twophase region based on their nuclear magnetic resonance (NMR) and X-ray diffraction studies of this transition. However, other experimental evidence showed that the $\delta-\mathrm{ZrH}_{x}(1.59 \leq x \leq 1.66)$ martensitically transforms to FCT $\varepsilon-\mathrm{ZrH}_{x}[15,20]$. As combination and summary of firstand second-order transformation findings, Moore et al. [22] concluded first order transition at the boundaries of the $\delta+\varepsilon$ two phase region, and second order transition at the boundary of the $\delta$ and $\varepsilon$ single phase regions with their high-temperature X-ray diffraction studies which was supplemented with dilatometric and electrical resis- tance measurements. According to [1], such discrepancies regarding the exact phase transformation order between $\delta$ and $\varepsilon$ might have been caused by the experimental difficulties in obtaining true equilibrium and also by the effects of oxygen impurities in the system. In addition, as many of these reported studies and structures are based on experiments carried out on ex-situ hydrided polycrystalline alloys or powders which crushed from hydrogenated alloys using different techniques and facilities, it is quite difficult to compare different measurements.

To identify the type of transition between $\delta$ - and $\varepsilon$-hydrides, we have performed an in-situ hydrogen charging experiment on beam line ID15-B at the European Synchrotron Radiation Facility (ESRF) in Grenoble, France. A high pressure/high temperature capillary system developed at the ESRF [23] was used to hydride $\mathrm{Zr}$ powder, and the phase transitions between $\delta$ - and $\varepsilon$-hdyrides was recorded in real time through one single setup. Rietveld [24] and Pawley [24] analyses were performed to determine the crystal structure of various phases.

\section{Materials and methods}

A commercial grade, pure $\mathrm{Zr}$ powder (99.2\% purity) with a maximum particle size about $45 \mu \mathrm{m}$ was obtained from Goodfellow Ltd., Huntingdon, England (ZR006015). Then, the $\mathrm{Zr}$ powder was filled into a sapphire container in an argon environment to prevent any sort of contamination or oxidation. To dissolve any hydrides that may have formed earlier or during preparation, the sealed cell which contain $\mathrm{Zr}$ powder were first baked under vacuum at $\sim 700{ }^{\circ} \mathrm{C}$ for $\sim 7.5$ hours, and then at $\sim 1000{ }^{\circ} \mathrm{C}$ 
for 5 hours until an ultra-high vacuum level was achieved.

\subsection{Hydrogen charging and data collection}

The angular dispersive diffraction setup available at ID15-B, with a high-energy X-ray beam $(87 \mathrm{keV})$ and large area detector (Trixell Pixium 4700, Thales), was used for data acquisition [23, 25].

In order to capture all transitions and ensure quality of the data during the hydrogenation $(\delta \rightarrow \varepsilon)$ and dehydrogenation $(\varepsilon \rightarrow \delta)$ process, $0.2 \mathrm{~s}$ and $20 \mathrm{~s}$ of data acquisition time were used, respectively. To ensure good diffraction signals from the specimens, $0.3 \times 0.3 \mathrm{~mm}$ beam size is selected for data collection. The wavelength of the $\mathrm{X}$ ray beam was calibrated with a standard $\mathrm{LaB}_{6}$ specimen (SRM 660a, lattice parameter $=4.1569162 \pm 0.0000097 \AA$ ) [26] to $\lambda=0.142352 \AA$ A . During the whole measurement, the wavelength was kept constant.

To determine the stability and formation mechanisms of the hydride phases, the measurement started at room temperature with pure $\mathrm{Zr}$ powder, and data were collected continuously throughout the measurement using the two acquisition time mentioned above. The heating and cooling rate were controlled to be $\sim 10{ }^{\circ} \mathrm{C} / \mathrm{min}$. Once the system temperature reached $300^{\circ} \mathrm{C}$, mixture of hydrogen and argon gas was introduced at 0.5 bar (hydrogen partial pressure). Then, the system was kept in these conditions for $3.5 \mathrm{~h}$ until no further phase transformation was observed. In order to study the reversibility of the reaction, the hydrogen and argon mixture supply were cut and temperature was raised until no more $\varepsilon$-hydride phase could be found in the system. After the completion of $\varepsilon \rightarrow \delta$ transformation, the temperature increase was continued and the $\alpha$-Zr phase started to form around $556{ }^{\circ} \mathrm{C}$. In the end, the temperature increase was stopped at $613{ }^{\circ} \mathrm{C}$ and the system cooled back to room temperature with the same rate.

\subsection{Data analysis}

The structure analysis software packages TopasAcademic [24] and GSAS [27] were used for the Rietveld and Pawley refinements. The basic crystal structure information of the various $\mathrm{Zr}$-hydride phases and pure $\mathrm{Zr}$ needed for both Rietveld and Pawley method was obtained from the literature [1].

To identify and confirm all existing phases at each distinct stable phase region from hydrogen charging and discharging, the Rietveld refinements were performed for a selected number of patterns in each region. As the system is not in equilibrium during the phase transformation, the Pawley refinements were carried out to follow the dynamics of the lattice parameter variation during phase transformation. In order to investigate the nature of the transformation, and possible coexistence of the two phases, the diffractograms around the transformation were fitted using two approaches, here referred to as the single phase approach, and the superposition approach, respectively.

Both Pawley and Rietveld refinements were performed on the full diffractogram which spans from 0.37 9.46 degrees in $2 \theta$ and correspondingly 21.71-0.86 ̊̊ in dspacing. The peak profiles were modeled with a modified Thompson-Cox-Hastings pseudo-Voigt (pV-TCHZ) profile function. The background was fitted with a Chebyshev function with nine coefficients and the zero shift error calibrated with the $\mathrm{LaB}_{6}$ reference specimen. As our material in question is a high purity, fine grained powder, we did not consider any preferred orientation or texture effects in our analysis. The fractional atomic coordinates were not refined at any time.

\subsubsection{The single phase approach}

After identification and confirmation of all existing phases at each distinct region, one pattern from a stable single-phase region, either $\varepsilon$ or $\delta$ depending on direction of transition refined, was selected and a Pawley refinement was performed upon that pattern. Using this Pawley fit from the well-defined $\varepsilon$ - or $\delta$-phase region as a starting point (c.f. $257-263 \mathrm{~min}$ is $\delta$-phase and $83-250$ $\min$ is for $\varepsilon$-phase in figure 1), sequential Pawley refinements were made for both dehydrogenation, $\varepsilon \rightarrow \delta$, and hydrogenation, $\delta \rightarrow \varepsilon$, direction. That is to say, only a single phase was used in the refinement for the whole transition region. This was motivated by the fact that upon visual inspection no coexistence of multiple phases was observed.

\subsubsection{The superposition approach}

On contrast to the single phase approach, in this method the refinement of each diffractograms in whole transition region contained both $\varepsilon$ - and $\delta$-phases. The crystal structure suggestion required for fitting procedure obtained from a selected pattern in well-defined single phase region as described in the single phase approach. Similar to the first approach, the least squares procedures were adopted for minimization the difference 

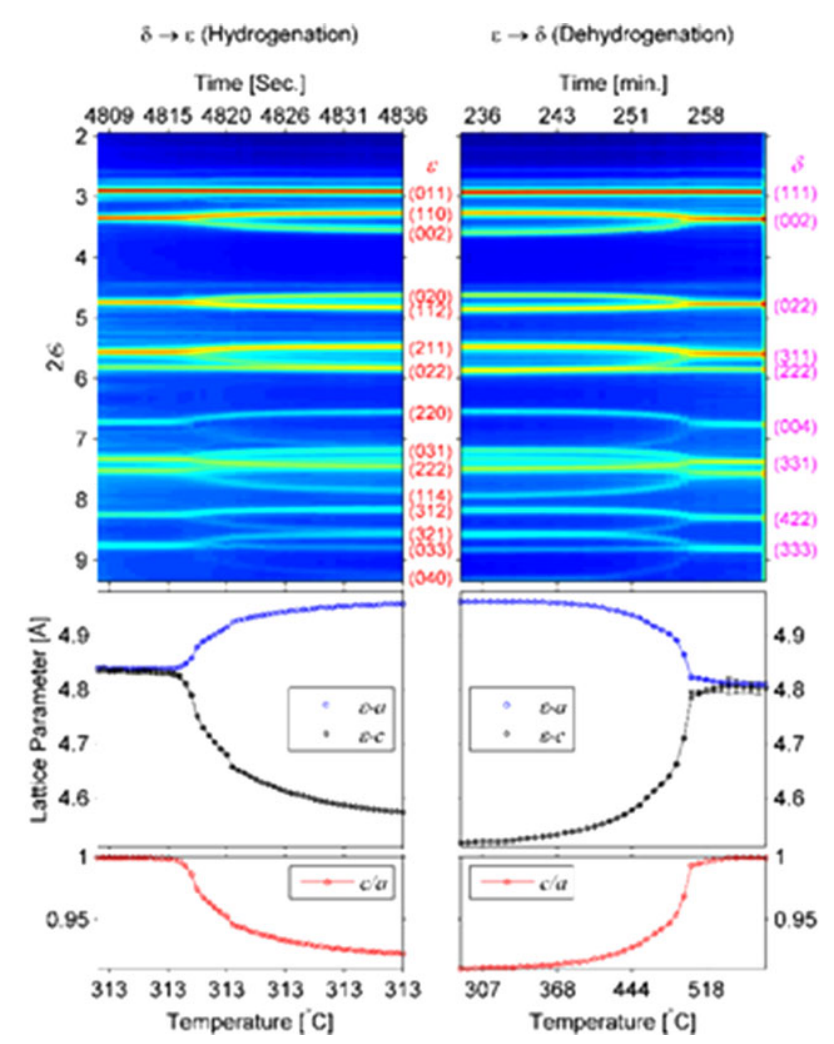

Fig. 1 Accumulated diffraction patterns between $\delta \leftrightarrow \varepsilon$ transformations together with their fitting results. The figures on right are from hydrogenation process and left are from reverse process. The figures in the middle are the Pawley fitting results of the corresponding diffractograms, where the error bars are mostly smaller than markers. The two figures at the bottom show the corresponding $\mathrm{c} / \mathrm{a}$ ratio. The intensity of both observed spectrum is plotted in log scale, and for clarity purpose some of the peak indices were not shown with text.

between the observed and simulated powder diffraction patterns and the minimization was carried out by using the reliability index parameter, $R_{w p}$ (weighted residual error), $R_{\exp }$ (expected error) and GoF (goodness of fit) [24, 27 . As the goodness of the various residual factors or ' $R$ factors' strongly correlated with number of free refinable parameters in the system, statistically it is always easier to get better R-factors in two phase system than the one phase despite the low quality of the fit graphically or unreasonable peak shapes of various involved single phases. Therefore, to avoid such refinement or other program related errors, in the superposition approach we had carefully controlled number of refinable parameters and their opening sequences. In addition, the quality of the fit and as well as the shape of the de-convoluted single phase peak profiles also investigated graphically. Despite our care, the superposition approach, however, did not succeed in producing reasonable results, e.g., the peak positions would not match to a satisfactory level. This confirmed the visual inspection, that no coexistence of phases could be determined. Hence, in the paper only the result of the first approach is presented.

\section{Results}

Two sections of the complete record collected at different time and temperatures during the hydrogenation and dehydrogenation process corresponding to $\delta \leftrightarrow \varepsilon$ transformation are shown in figure 1 as a two-dimensional contour plot (two figures on top), with time and temperature along the abscissa and peak positions along the ordinate. Note, that all the data in figure 1 were recorded in single measurement. Most of the HKL peak indices of the $\delta$ and $\varepsilon-\mathrm{ZrH}_{x}$ are shown at corresponding Bragg positions. Although there are visible traces of $\mathrm{Zr}$-oxide in some spectrums, for clarity their indices were not shown in this or other figures in the paper. From figure 1 one can clearly identify the hallmarks of the FCC $\leftrightarrow$ FCT transformation as the $\{111\}$ and $\{222\}$ reflections remain unsplit, while the $\{002\},\{022\}$ and $\{331\}$ reflections each split into two lines.

Extracted diffraction patterns at different transition time are shown in figure 2 from bottom to top. From figure 2 it can be seen that in the beginning of hydrogen charging there was only pure $\alpha-\mathrm{Zr}$ phase. After introducing hydrogen gas at $313^{\circ} \mathrm{C}$ the $\alpha$-Zr phase quickly transformed into $\delta-\mathrm{ZrH}_{x}$ phase. Then, the $\delta-\mathrm{ZrH}_{x}$ transformed into hydrogen rich FCT $\varepsilon-\mathrm{ZrH}_{x}$. This whole transition from $\alpha-\mathrm{Zr}$ phase to $\varepsilon-\mathrm{ZrH}_{x}$ phase was completed in a matter of seconds. After the completion of $\delta$ to $\varepsilon$ transition no further structural change was observed for

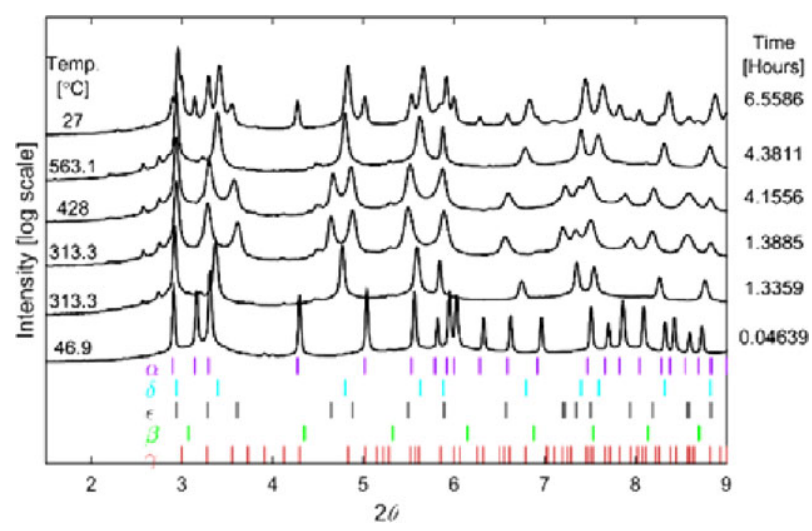

Fig. 2 Selected diffraction patterns. The color coded, small vertical tick marks represent the HKL peak positions of the labelled phases. The small unindexed peaks correspond to $\mathrm{Zr}$-oxide peaks. 


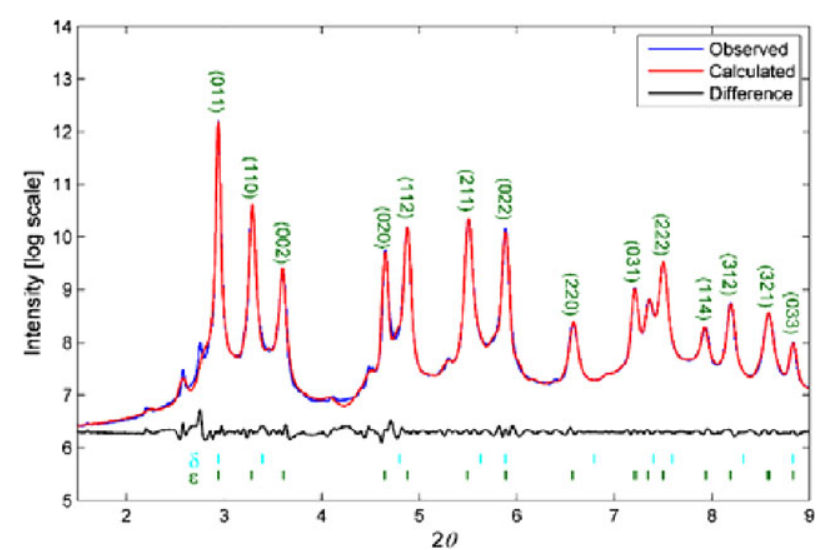

Fig. 3 One typical Pawley refinement result: $R_{w p}$ 6.549, GOF 1.762 .

about an hour. To investigate the reversibility of this transition, we raised the temperature to $613{ }^{\circ} \mathrm{C}$ and degassed the system. Following the temperature increase, the hydrogen rich $\varepsilon-\mathrm{ZrH}_{x}$ transforms back to cubic $\delta-\mathrm{ZrH}_{x}$ at a temperature of approximately $518{ }^{\circ} \mathrm{C}$ with an identical but reverse transformation sequence and corresponding diffraction patterns as observed previously during the $\delta$ to $\varepsilon$ transformation (figure 1 ).

Figure 3 represents a typical Pawley refinement result within the $\varepsilon$ phase region at $313^{\circ} \mathrm{C}$ seen in figure 1 . In the figure the blue solid line represents observed intensities, the red line represents the calculated, and the black line is the difference curve on the same scale. The tick marks indicate the calculated positions of Bragg peaks. As seen from the difference curve, the fit is highly satisfactory.

Some of the unit cell parameters obtained by the Rietveld refinement on selected data from each distinct phase region are compared with previously published data [1] in Table 1. The lattice parameter variations during the hydrogenation and dehydrogenation processes obtained from the Pawley refinements are presented in figure 1. The calculation of the Pawley refinement started from $\varepsilon$ phase region with $\varepsilon$ phase unit cell parameters mentioned in the introduction and ended in the $\delta$ phase region with an $\varepsilon$ space group and lattice parameters. As seen from the figure, during hydrogen loading process the $\varepsilon-\mathrm{ZrH}_{x}$ lattice parameter $a$ increased while $c$ and $c / a$ ratio decreased steadily with increasing hydrogen concentration. The inverse transformation phenomenon also can be seen during hydrogen unloading from both fitting results and as well as transformation maps in figure 1.

The tetragonality of the $\varepsilon$ phase first gradually increases then decreases with hydrogen concentration as shown in figure 1 . The $c / a$ ratio of the $\varepsilon$ phase starts from one and slowly reaches to approximately 0.9 , then slowly approaches back to unity again corresponding to hydrogen in and out direction with the implication of cubic to tetragonal and tetragonal to cubic transition. The structural change between $\delta \rightarrow \varepsilon$ and $\varepsilon \rightarrow \delta$ are smooth and there is no discontinuity observed in figure 1 or figure 4. Apart from the continuous change of the lattice parameters of $\delta$ and $\varepsilon$ phases during the $\delta \leftrightarrow \varepsilon$ transition, no coexistence of these two phases has been observed. These results justify the assumption that the crystal structure of $\varepsilon$ and $\delta$ continuously adapt from tetragonal space group I $4 / \mathrm{mmm}$ to cubic Fm-3m depending on temperature and hydrogen concentrations. This result are in line with Yakel's [28] observavtion for $\mathrm{TiH}_{2}$ which is chemically and crystallographically very similar to $\mathrm{ZrH}_{2}$.

In order to clarify smooth variation between $\delta$ and $\varepsilon$, the diffractograms collected near $\delta \rightarrow \varepsilon$ and $\varepsilon \rightarrow \delta$ transformations are shown in figure 4 as a waterfall plot. As shown in the figure, the characteristic peaks split and merge continuously during hydrogen charging and discharging. Here it should be noticed that during the hydrogenation stage the temperature remained constant while the hydrogen concentration varies, but during the dehydrogenation stage we recorded both varying temperature and hydrogen concentration. Obtaining complete temperature stability during discharging is experimentally challenging. This transformation behaviour is a good indication of the transformation order being $2^{\text {nd }}$ order.

\section{Discussion}

The lattice parameters of the $\alpha$ - Zr phase obtained in this study $(a=3.24205 \AA$ and $c=5.16645 \AA$ ) are in good agreement with reported values [1]. It should be noted that neither our experiment nor that reported in literature was aimed at absolute measurement of the lattice parameter. During the initial heating and prior to hydrogen loading, the $\alpha$-Zr phase showed linear thermal expansion behavior with respect to temperature. After cooling from $613{ }^{\circ} \mathrm{C}$ to room temperature, the final $\alpha$ - $\mathrm{Zr}$ lattice was within $<1 \%$ of the initial lattice parameter. It should be noted here that the lattice parameters depends in a very sensitive manner on the sample to detector distance and may have moved. We never intended to determine absolute lattice parameter values, but only indicate the relative changes. As shown in figure 2 , at the end of the heating cycle both $\alpha-\mathrm{Zr}$ phase and $\delta-\mathrm{ZrH}_{x}$ phase are found in the system, and according to our Rietveld analysis the amount of $\delta$-hydride phase is quite substantial (81.94 wt\%). Therefore, it is not surprising to see such small 


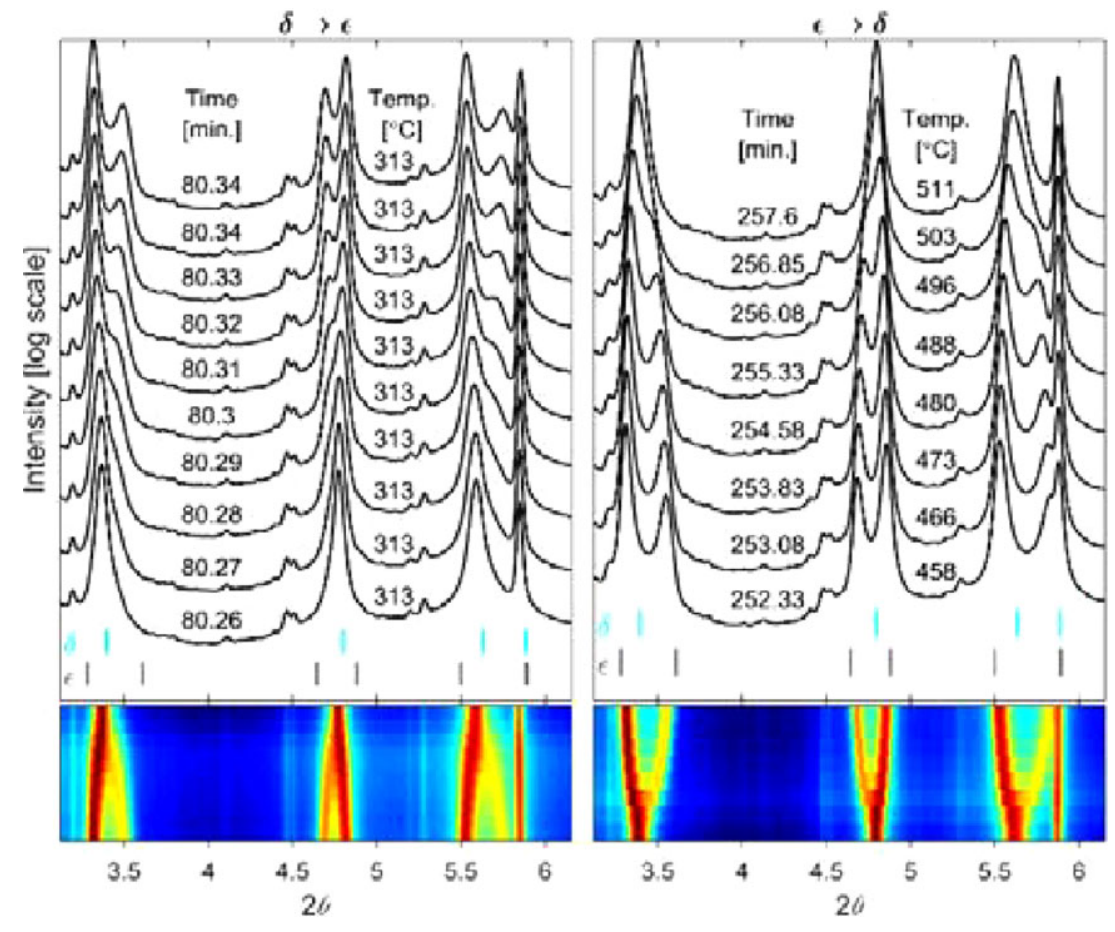

Fig. 4 The parts of diffractograms collected near $\delta \leftrightarrow \varepsilon$ transformation.

variation of $\alpha$-Zr lattice parameter before and after the treatment. The lattice parameters of $\delta-\mathrm{ZrH}_{x}$ before $\delta \rightarrow \varepsilon$ transformation showed larger values than the reported for corresponding measurement temperature but agreed very well with the data published in the literature [1]. The possible cause of such $\delta-\mathrm{ZrH}_{x}$ lattice parameter difference prior to transformation can be explained by the sensitivity of the lattice parameter determined by diffraction on the sample to detector distance, which may have changed during the charging.

The order of the observed phase transformation can be classified based on the variation of thermodynamic parameters, phase formation mechanism or transformation kinetics [29]. For diffraction, the phase transition order parameters can be defined by variation in atomic concentration of composing atoms of the phase or by unit cell parameters, such as: $a, c, V, a / c, \Delta l / \Delta T$ where $l$ is $a$ or $c[19,22,28]$. If any one of these parameters shows a continuous change with respect to a temperature or composition variation, the transition is therefore likely to be a second-order transformation. However, if two phases coexist at the boundary (a nucleation and growth type transition) of a transformation, the transition can be considered as first-order disregarding the continuous variation of other lattice parameters. The classic martensitic transformation is a displacive, first-order transition and the unit cell dimensions typically show composition dependence [19]. In diffraction experiments, the martensitic transition is visible through the appearance of second phase Bragg peaks at the transition point.

From the diffraction map in in figure 1 and diffractograms in figure 4 , it is evident that the variations from one phase to another is rather smooth and there is no sudden change of any existing peak positions, or sudden appearance or disappearance of new phase peaks as for first order or martensitic transformation. Moore et al. [22] also observed similar results for $\varepsilon \rightarrow \delta$ in $\mathrm{Zr}$ and Yakel [28] in Ti. The variations of lattice parameter $a, c$ and $c / a$ ratio between FCC and FCT are smooth, and unit cell dimensions are hydrogen concentration depended. This kind of transformation behaviours are consistent with second-order phase transformations as described in [19, 22, 28]. Additionally, trying to fit $\delta \leftrightarrow \varepsilon$ transition region using superimposition of both pure $\delta$ and pure $\varepsilon$ phases did not give any reasonable results with indication that there is no coexisting $\delta$ - and $\varepsilon-\mathrm{ZrH}_{x}$ in the system. The $c / a$ ratio and unit cell parameter $c$ of $\varepsilon-\mathrm{ZrH}_{x}$ presented in figure 1 clearly shows hysteresis between hydrogen charging and discharging. However, it should be realized that there is a hydrogen concentration difference between these two stages. As concentration of hydrogen just before dehydrogenation is relatively larger than just after completion of $\delta \rightarrow \varepsilon$, the unit cell of $\varepsilon-\mathrm{ZrH}_{x}$ appears than the lower concentrated counterpart, but we cannot claim this with full confidence, as the determined 
lattice parameter is very sensitive to minute changes in sample position.

From figure 4, one can clearly see that there is no evidence of secondary Bragg peaks during the entire $\delta \leftrightarrow \varepsilon$ transformation range. As shown, all Bragg peaks continuously split or merge depending on the direction of transformation. This is in agreement with the discussion above, and in $[15,30]$, applying the phenomenology of martensitic transformation to this transition, which, based on hydrogen diffusion, cannot be purely displacive (because it relies on the diffusion of hydrogen) but of a similar nature as the bainitic transformation.

Both [1] and [22] stated that because of narrow twophase $(\delta+\varepsilon)$ region, and influence of oxygen as well as some other impurities in the $\mathrm{Zr}-\mathrm{H}$ system, it is very difficult to establish a firm relation between unit cell dimensions and hydrogen composition in this region. According to [22], oxygen concentrations over 3.5 at\% will significantly affect the boundaries of the $\delta+\varepsilon$ region, pushing it towards lower $\mathrm{H} / \mathrm{Zr}$ ratios, while the region also becomes wider with increasing oxygen concentration. However, while we have small amounts of oxygen in our system and while our hydrogen loading temperature is $310^{\circ} \mathrm{C}$ which is significantly below the reported twophase region around a temperature $550{ }^{\circ} \mathrm{C}$, we did not observe any coexistence of $\delta$ and $\varepsilon$ phases in our measurements. Thus, we can presume that the transformation order between $\delta$ and $\varepsilon$ might belong to the secondorder transformation type. Here, it should be noted that we do not exclude the possibility of $\delta$ and $\varepsilon$ coexistence at other concentrations or temperatures. According to other measurements performed by is, which are not reported here for the sake of brevity, and according to literature [31], these two phases have been observed simultaneously, e.g. at room temperature. From figure. 1 presented in [31], one can conclude that it is common to have inhomogeneity of hydrogen distribution in the $\mathrm{Zr}$ alloys after hydrogenation in particular at lower temperatures with less hydrogen mobility.

Phenomenologically, one can consider an order parameter $\beta$ based on the normalized hydrogen concentration with respect to $\varepsilon-\mathrm{ZrH}_{x}$, such as $\beta=(2-x) / 2$, where $x$ is the $\mathrm{H} / \mathrm{Zr}$ ratio during the transition, in the range of 1.66-2. According to this definition the order parameter, at least close to this transition region, will take values in interval $[0.17,1]$ depending on concentration of hydrogen atoms. When all eight tetrahedral positions are completely filled with hydrogen in hydride unit cell the $\beta=0$, and $\beta=1$ in the extreme case when all sites are empty (no hydrogen atoms). Of course, the authors are aware, that this order parameter is not valid for any assumed $\gamma$ $\delta$ transition at concentrations less than $x=1.66$.

\section{Conclusions}

For the first time, in-situ hydrogen charging studies were performed on high purity Zr powder in order to identify phase stability and the type of transformation between $\delta$ and $\varepsilon$ Zr-hydride.

A transformation between $\delta$ and $\varepsilon$ was recorded with a single experiment, where the determined lattice parameters of $\alpha-\mathrm{Zr}$ phase and $\delta-\mathrm{ZrH}_{\mathrm{x}}$ are in good agreement with values reported in the literature. Upon charging at $310^{\circ} \mathrm{C}$ the $\delta$-phase transform smoothly into hydrogen rich $\varepsilon$ $\mathrm{ZrH}_{x}$. No coexistence of $\delta$ and $\varepsilon$ was observed. Upon degassing at elevated temperatures $\left(500-600^{\circ} \mathrm{C}\right)$, the hydrogen rich $\varepsilon$-phase transforms back to first pure $\delta$-phase and then $\alpha-\mathrm{Zr}+\delta-\mathrm{ZrH}_{x}$ as a function of $\mathrm{H} / \mathrm{Zr}$ ratio.

The observed phase transformation between $\varepsilon$ and $\delta$ showed characteristics of a second order transformation, and hydrogen occupancy is suggested as an order parameter in the transformation region.

Acknowledgements. The ESRF is gratefully acknowledged for the provision of beam time under MA-1371. We are thankful to the Swedish Research Council, Vetenskapsrådet, for financial support via grant No. VR 2008-3844. We acknowledge in-depth discussions with Dr. JE Daniels at the University of New South Wales, Australia.

Key words. Synchrotron X-ray diffraction, Zirconium hydride, phase transformation.

\section{References}

[1] E. Zuzek, J. Abriata, A. San-Martin, and F. Manchester, Bulletin of Alloy Phase Diagrams 11, 385 (1990).

[2] A. Steuwer, J. R. Santisteban, M. Preuss, M. J. Peel, T. Buslaps, and M. Harada, Acta. Mater. 57, 145 (2009).

[3] P. A. T. Olsson, A. R. Massih, J. Blomqvist, A. M. Alvarez Holston, and C. Bjerkén, Comput. Mater. Med. 86, 211 (2014).

[4] L. Lanzani and M. Ruch, Journal of Nuclear Materials 324, 165 (2004).

[5] R. N. Singh, S. Mukherjee, A. Gupta, and S. Banerjee, J. Alloys. Compd. 389, 102 (2005).

[6] C. E. Coleman and D. Hardie, J. Less Common Metals 2, 168 (1966).

[7] D. O. Northwood and U. Kosasih, Int. Mater. Rev. 28, 92 (1983).

[8] IAEA. International Atomic Energy Agency (IAEA), IAEA TECDOC 1649 (2010)

[9] Z. Zhao, J. P. Morniroli, A. Legris, A. Ambard, Y. Khin, L. Legras et al., J. Microsc. 232, 410 (2008).

[10] M. Kerr, PhD thesis, Mechanical and Materials Engineering, Queen's University 2009.

[11] E. Tulk, M. Kerr, and M. R. Daymond, J. Nucl. Mater. 425, 93 (2012). 
[12] J. H. Root, W. M. Small, D. Khatamian, and O. T. Woo, Acta. Mater. 51, 2041 (2003).

[13] A. I. Kolesnikov, A. M. Balagurov, I. O. Bashkin, and A. V. Belushkin, J. Phys.: Condens. Matter 6, 8977 (1994).

[14] R. C. Bowman, J. E. L. Venturini, B. D. Craft, A. Attalla, and D. B. Sullenger, Phys. Rev. B 27, 1474 (1983).

[15] M. P. Cassidy and C. M. Wayman, Metallogr. and Mater. Trans. A 11, 57 (1980).

[16] B. W. Veal, D. J. Lam, and D. G. Westlake, Phys. Rev. B 19, 2856 (1979).

[17] W. M. Mueller, J. P. Blackledge, and G. G. Libowitz, Metal hydrides. (California: Academic Press, 1968).

[18] V. I. Ivashchenko, L. A. Ivashchenko, P. L. Srynsckyy, L. A. Grishnova, and A. I. Stegnyy. Ab Initio Study of the Electronic Structure and Phonon Dispersions For TiH2 and ZrH2. In: B. Baranowski, S. Zaginaichenko, D. Schur, V. Skorokhod, and A. Veziroglu Editors. Carbon Nanomaterials in Clean Energy Hydrogen Systems, (Springer Netherlands, 2009). p. 705.

[19] J. S. Cantrell, R. C. Bowman Jr., and D. B. Sullenger, J. Phys. Chem. 88, 918 (1984).
[20] K. G. Barraclough and C. J. Beevers, J. Nucl. Mater. 34, 125 (1970).

[21] K. E. Moore, J. Nucl. Mater. 32, 46 (1969).

[22] K. E. Moore and W. A. Young, J. Nucl. Mater. 27, 316 (1968).

[23] J. Andrieux, C. Chabert, A. Mauro, H. Vitoux, B. Gorges, T. Buslaps et al., J. Appl. Crystallogr. 47, 245 (2014).

[24] A. Coelho, Topas Academic: Technical Reference 2004;V4.1:http://www.topas-academic.net/.

[25] J. E. Daniels, J. Appl. Crystallogr. 41, 1109 (2008).

[26] W. F. Stephen and M. T. Nancy, NIST Standard Reference Materials: MD 20899, (2000).

[27] A. C. Larson and R. B. Von Dreele, Los Alamos Natl. Lab. Rep. LAUR, 86 (2000).

[28] J. H. L. Yakel, Acta. Crystallogr. 11, 46 (1958).

[29] D. A. Porter and K. E. Easterling, Phase Transformations in Metals and Alloys. (Springer US, 1992).

[30] M. P. Cassidy and C. M. Wayman, Metallogr. and Mater. Trans. A 11, 47 (1980).

[31] R. S. Daum, Y. S. Chu, and A. T. Motta, J. Nucl. Mater. 392, 453 (2009). 